\title{
Relativistic quantum tunneling of a Dirac fermion in nonhyperbolic chaotic systems
}

\author{
Xuan $\mathrm{Ni}^{1}{ }^{1}$ Liang Huang,${ }^{1,2}$ Lei Ying, ${ }^{1}$ and Ying-Cheng Lai ${ }^{1,3, *}$ \\ ${ }^{1}$ School of Electrical, Computer and Energy Engineering, Arizona State University, Tempe, Arizona 85287, USA \\ ${ }^{2}$ Institute of Computational Physics and Complex Systems, and Key Laboratory for Magnetism and Magnetic Materials of MOE, \\ Lanzhou University, Lanzhou, Gansu 730000, China \\ ${ }^{3}$ Department of Physics, Arizona State University, Tempe, Arizona 85287, USA
}

(Received 17 April 2013; revised manuscript received 29 May 2013; published 19 June 2013)

\begin{abstract}
Nonhyperbolicity, as characterized by the coexistence of Kolmogorov-Arnold-Moser (KAM) tori and chaos in the phase space, is generic in classical Hamiltonian systems. An open but fundamental question in physics concerns the relativistic quantum manifestations of nonhyperbolic dynamics. We choose the mushroom billiard that has been mathematically proven to be nonhyperbolic, and study the resonant tunneling dynamics of a massless Dirac fermion. We find that the tunneling rate as a function of the energy exhibits a striking "clustering" phenomenon, where the majority of the values of the rate concentrate on a narrow region, as a result of the chaos component in the classical phase space. Relatively few values of the tunneling rate, however, spread outside the clustering region due to the integrable component. Resonant tunneling of electrons in nonhyperbolic chaotic graphene systems exhibits a similar behavior. To understand these numerical results, we develop a theoretical framework by combining analytic solutions of the Dirac equation in certain integrable domains and physical intuitions gained from current understanding of the quantum manifestations of chaos. In particular, we employ a theoretical formalism based on the concept of self-energies to calculate the tunneling rate and analytically solve the Dirac equation in one dimension as well as in two dimensions for a circular-ring-type of tunneling systems exhibiting integrable dynamics in the classical limit. Because relatively few and distinct classical periodic orbits are present in the integrable component, the corresponding relativistic quantum states can have drastically different behaviors, leading to a wide spread in the values of the tunneling rate in the energy-rate plane. In contrast, the chaotic component has embedded within itself an infinite number of unstable periodic orbits, which provide far more quantum states for tunneling. Due to the nature of chaos, these states are characteristically similar, leading to clustering of the values of the tunneling rate in a narrow band. The appealing characteristic of our work is a demonstration and physical understanding of the "mixed" role played by chaos and regular dynamics in shaping relativistic quantum tunneling dynamics.
\end{abstract}

DOI: $10.1103 /$ PhysRevB.87.224304

PACS number(s): 05.45.Mt, 03.65.Pm, 73.63.-b

\section{INTRODUCTION}

A challenging and fundamental problem in the field of quantum chaos ${ }^{1}$ is to explore and understand quantum manifestations of chaotic behaviors in situations where the classical dynamics is nonhyperbolic. In classical Hamiltonian systems, nonhyperbolicity is characterized by the coexistence of chaos and Kolmogorov-Arnold-Moser (KAM) tori in the phase space, hence the term "mixed phase space."2-4 The problem has been addressed previously but mostly in the realm of nonrelativistic quantum mechanics governed by the Schrödinger equation. For example, in open Hamiltonian systems where the classical dynamics is chaotic scattering, the presence of KAM tori can result in enhanced fluctuations of the semiclassical $S$-matrix elements ${ }^{5}$ or even lead to fractal fluctuations. ${ }^{6}$

Development of graphene $e^{7,8}$ physics in the past decade stimulated a great deal of interest in relativistic quantum mechanics as applied to solid-state devices. Graphene systems, such as quantum dots that exhibit chaos in the classical limit, have been studied with respect to issues such as the energylevel statistics, quantum scars, and scattering dynamics. ${ }^{9}$ From the classical point of view, nonhyperbolic systems are generic, while integrable and fully chaotic systems are the exceptions, as the latter correspond to the two opposite extreme cases in the spectrum of nonlinear Hamiltonian systems. Thus, in applications of graphene devices, nonhyperbolic dynamics is expected to be more common than integrable or chaotic dynamics, and this demands a good understanding of the relativistic quantum manifestations of nonhyperbolicity.

The linear energy-momentum relation in graphene systems, which is characteristic of relativistic quantum motion, however, holds only approximately near the Dirac points in the energy-band diagram. In addition, while the motion of electrons in graphene near a single Dirac point can be described by the Dirac equation, ${ }^{8}$ in realistic systems the coupling between motions associated with the coexisting Dirac points cannot be neglected. Thus, strictly speaking, the study of chaotic graphene systems can lead only to a partial understanding of various phenomena in the emergent field of relativistic quantum chaos. To obtain a complete picture, fermion systems governed by the Dirac equation must be studied. In this regard, the manifestation of classical chaos in a strictly relativistic quantum system, the neutrino billiard, was first studied by Berry and Mondragon, ${ }^{10}$ who developed a boundary-integral method to calculate the eigenvalues and eigenvectors of the zero-mass Dirac equation in two-dimensional chaotic domains. Quite recently, a conformal-mapping based method with great computational efficiency has been developed for the Dirac equation, revealing the existence of a class of "chiral" scars ${ }^{11}$ with unusual phase properties. The relativistic quantum tunneling dynamics has also been studied. ${ }^{12}$ However, in all these works, the underlying classical system is fully chaotic, 

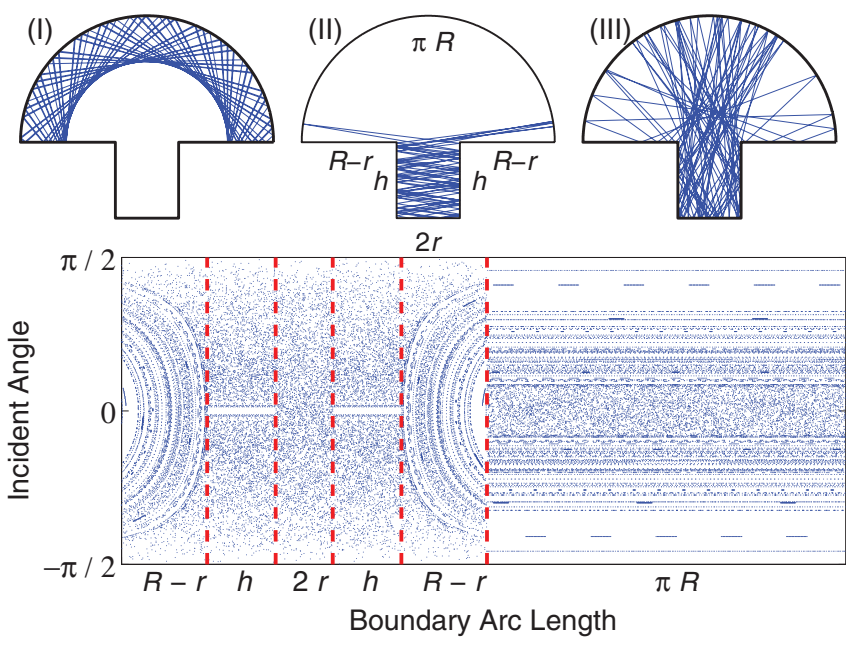

FIG. 1. (Color online) Classical trajectories and phase map (billiard map) of a mushroom billiard that consists of a semicircular cap and a rectangular stem. The top row shows three different types (I-III) of classical trajectories: stable and marginally stable periodic, as well as chaotic orbits. The bottom panel depicts the phase map in the phase space defined by the boundary arc length and the incident angle. The regions separated by the red vertical dashed lines correspond to the line/arc boundary segments marked in (II).

or hyperbolic in which all periodic orbits are unstable. To our knowledge, there has been no prior work on the relativistic quantum manifestations of nonhyperbolic chaotic systems.

A paradigm of nonhyperbolic chaotic systems is the "mushroom" billiard for which a mathematical proof of nonhyperbolicity was obtained by Bunimovich ${ }^{13}$ A representative mushroom billiard is shown in the top row of Fig. 1, together with some typical orbits. Nonhyperbolicity with a mixed phase space can be seen in the bottom panel that depicts the phase map of the incident angle versus the arc length along the mushroom boundary. By placing a finite potential barrier along the vertical, symmetric line of the billiard, we effectively generate the setting of resonant tunneling where a particle can tunnel through the barrier from one side of the billiard to the other. We assume that the particle is a massless Dirac fermion, whose motion is governed by the Dirac equation, and we focus on the effect of nonhyperbolicity on the tunneling rate $\gamma(E)$, the fundamental quantity characterizing the quantum tunneling dynamics, which depends on the particle energy $E$. Our main finding is that nonhyperbolicity leads to a "clustering" phenomenon where the majority of the values of $\gamma(E)$ fall into a narrow "band" in the $(E, \gamma)$ plane, with relatively few values outside the band. To understand this clustering phenomenon, we develop a theoretical framework based on the concept of self-energies to calculate the tunneling rate. We then analytically solve the Dirac equation both in one and in two dimensions for a circular-ring type of tunneling systems with integrable dynamics in the classical limit. Due to the presence of relatively few and distinct classical periodic orbits in the integrable component, the corresponding relativistic quantum states can have drastically different behaviors, leading to a wide spread of the values of the tunneling rate in the $(E, \gamma)$ plane. However, the chaotic component provides significantly more quantum states for tunneling due to the infinite set of unstable periodic orbits embedded therein. These states are dynamically "similar" because of the ergodic nature of chaos, and this leads to the clustering of the values of the tunneling rate in a narrow band.

We remark that there were recent works on nonrelativistic quantum tunneling in nonhyperbolic chaotic systems but in the phase space such as the tunneling from regular KAM islands to the surrounding chaotic sea. ${ }^{14}$ While we also deal with nonhyperbolic systems, the setting of our problem is resonant tunneling in the physical space in relativistic quantum systems.

In Sec. II, we describe the classical dynamics of the mushroom-billiard system, discuss our method to solve the Dirac equation in an arbitrary closed domain, and present an approach to calculating the quantum tunneling rate. In Sec. III, we present numerical results of the clustering phenomenon in the rate-energy plane for both Dirac fermion and graphene systems. In Sec. IV, we develop a theoretical framework, combining analytic results from solutions of the Dirac equations in certain integrable domains and physical insights, to understand the numerical findings. Concluding remarks are offered in Sec. V.

\section{THE MUSHROOM BILLIARD: NONHYPERBOLIC CLASSICAL DYNAMICS, SOLUTIONS OF THE DIRAC EQUATION, AND THE TUNNELING RATE}

\section{A. Nonhyperbolic dynamics in the classical limit}

The mushroom billiard exhibits a mixed phase space and the distribution of the recurrence time is algebraic, a typical characteristic of chaotic Hamiltonian systems. ${ }^{3,4}$ It is one of the very few Hamiltonian systems for which these features can be proven rigorously, ${ }^{13}$ and the corresponding nonrelativistic quantum tunneling dynamics has been studied. ${ }^{15}$ As shown in the top row of Fig. 1, in the classical limit, there are stable and marginally stable periodic orbits, as well as chaotic orbits. To generate a phase space, we note that the dynamics of the collision follows the simple rule of reflection. A discrete-time billiard map can then be derived where two dynamical variables, the boundary arc length from the left corner of the mushroom cap and the incident angle at a collision point, can be used to characterize the trajectories, as shown in the bottom panel of Fig. 1. One can identify two regions in the physical space responsible for the integrable dynamics: one corresponding to the semicircle $\pi R$ and the two boundary segments $R-r$ in which there are periodic orbits around the mushroom cap (type-I orbit in Fig. 1), and another located within the two boundary segments $h$ for small incident angles (type-II marginally stable periodic orbit in Fig. 1). The area of the latter in the billiard map increases with the length of the mushroom stem. When the incident angle becomes sufficiently large on the stem part (or small on the cap part) of the boundary, the trajectories are chaotic, covering both parts of the mushroom, as shown as the type-III orbit in Fig. 1.

\section{B. Numerical solutions of the Dirac equation}

The Dirac equation for massless fermion reads

$$
[v(\boldsymbol{\sigma} \cdot \boldsymbol{p})+V] \psi=E \psi,
$$


where $v$ is the Fermi velocity (e.g., $\sim 10^{6} \mathrm{~m} / \mathrm{s}$ for graphene), $\boldsymbol{\alpha}=\boldsymbol{\sigma}=\left(\sigma_{x}, \sigma_{y}\right)$ are the Pauli matrices, and $V=V(x, y)$ is the potential function. We solve the equation in the mushroom domain by using the efficient finite-difference method that we have recently developed, ${ }^{17}$ with a proper treatment of the boundary conditions and a strategy to overcome the Fermion doubling effect. In particular, the fact that the solutions of the massless Dirac equation are nonlocal ${ }^{16}$ has long been recognized as an obstacle to using the Dirac equation as a quantum mechanical framework. The problem of this nonlocalization is rooted in both the boundary treatment of the massless Dirac equation in a confined domain as well as the spatial discretization in the numerical solution. Instead of letting the wave function be zero as in a typical confined, nonrelativistic quantum system, one must set zero the outgoing current flux for a massless Dirac fermion. While straightforward discretization of the space will cause the massless Dirac equation to have localized solutions, a nonlocalized solution requires that the Dirac spinor and the Dirac equation be numerically evaluated at different locations in the space. The method ${ }^{17}$ allows us to obtain a large number of eigenvalues and the associated eigenstates for the whole closed domain.

\section{Method to calculate the relativistic quantum tunneling rate}

Throughout this paper, we use the tunneling rate to quantify the underlying relativistic quantum tunneling dynamics. For a symmetric closed domain with a potential barrier located along the line of symmetry so that the subdomains on both sides of the barrier are effectively potential wells, the tunneling rate characterizes how fast a quantum particle can tunnel through the potential barrier, in a probabilistic sense. Specifically, suppose that initially, the probability of finding the particle in one well is unity. The tunneling rate is determined by the inverse of the time required to observe a substantial fraction of the probability in the other well.

For nonrelativistic quantum tunneling, due to the geometrical symmetry in the double-well system, the eigenstates are either symmetric or antisymmetric about the central barrier. A symmetric/antisymmetric pair means that, on one side of the double well, the eigenfunctions completely overlap, while on the other side, the eigenfunctions differ by the factor of -1 . The tunneling rate is thus given by the energy splitting $\Delta E$ between the symmetric and antisymmetric eigenstate pairs ${ }^{18}$ which can be seen, as follows. Suppose we have symmetric and antisymmetric eigenstate pairs $\psi_{S}$ and $\psi_{A}$. Define a new state $\psi(t)=\psi_{S} e^{-i E_{S} t / \hbar}+\psi_{A} e^{-i E_{A} t / \hbar}$. At time $t=0, \psi(0)=\psi_{S}+\psi_{A}$ describes a state in which the particle can be found only on one side of the double well. We let this state evolve and find that at time $t_{0}=\pi \hbar / \Delta E$, where $\Delta E=\left|E_{S}-E_{A}\right|, \psi\left(t_{0}\right) \propto \psi_{S}-\psi_{A}$, the particle has tunneled to the other side of the well.

For Dirac fermion or graphene systems, such an energy splitting cannot be defined in general, for the following two reasons. (1) There is no clear criterion to separate symmetric/antisymmetric pairs from other mixed states. That is, a symmetric state cannot necessarily be paired with a corresponding antisymmetric state. (2) For a massless Dirac fermion, because of the violation of the time-reversal symmetry, ${ }^{10}$ the reflection symmetry is also broken. In par- ticular, due to the derivative relation of the two components of the spinor wave function from the Dirac equation (1), if one component of the spinor eigenstate is symmetric, the other component will be antisymmetric. As a result, the whole spinor eigenstate itself does not have to be symmetric or antisymmetric. We thus see that, for relativistic quantum tunneling systems, a new and more general definition of the tunneling rate is needed. A practical method is to construct a special class of initial states, states that concentrate on one side of the potential barrier only, and to monitor their time evolution. The time it takes for the probability concentration to switch between the two sides of the barrier is proportional to the tunneling rate.

Our approach is the following. ${ }^{12}$ For an arbitrary symmetric double-well system, we pick a random linear combination of eigenstates denoted as $\psi=\sum_{n} a_{n} \psi_{n}(\boldsymbol{r})$, where it is not necessary to run $n$ over all eigenstates, assuming that the eigenstates can be solved for any closed geometry. We then keep only the left side of $\psi$ and set the right side and the barrier part of $\psi$ to zero. We renormalize this state and denote it as $\bar{\psi}=$ $\sum_{n} \bar{a}_{n} \psi_{n}^{L}(\boldsymbol{r})$, where $\bar{a}_{n}$ 's are the renormalized coefficients, and $\psi_{n}^{L}(\boldsymbol{r})=\psi_{n}(\boldsymbol{r})$ for $\boldsymbol{r}$ in the left well, and $\psi_{n}^{L}(\boldsymbol{r})=0$ otherwise. Next, we let this state $\bar{\psi}$ evolve with time. It is necessary to express it in terms of a linear combination of all eigenstates, $\bar{\psi}=\sum_{m} b_{m} \psi_{m}(\boldsymbol{r})$, where the summing index $m$ runs through all eigenstates. The coefficients $b_{m}$ can then be calculated as

$$
\begin{aligned}
b_{m} & =\int_{D} \psi_{m}^{*} \bar{\psi} d \boldsymbol{r}=\int_{L} \psi_{m}^{*} \sum_{n} \bar{a}_{n} \psi_{n}^{L} d \boldsymbol{r} \\
& =\sum_{n} \bar{a}_{n} \int_{L} \psi_{m}^{*} \psi_{n} d \boldsymbol{r},
\end{aligned}
$$

where $D$ and $L$ denote the integration domains of the whole double well and of the left well, respectively. The time evolution of the state $\bar{\psi}$ is then given by $\bar{\psi}(t)=\sum_{m} b_{m} \psi_{m} e^{-i E_{m} t / \hbar}$. Because the particle state is initially confined within the left well, to characterize the tunneling process of this state, we calculate the probability that the particle is found in the left well with respect to time, $P_{L}(t)=\int_{L}|\bar{\psi}(t)|^{2} d \boldsymbol{r}$. Typically, the tunneling rate $R$ is found at the time when $P_{L}(t)$ reaches minimum for the first time, i.e.,

$$
R \sim 1 / \Delta T,
$$

where the proportional constant can be determined using simple analyzable models (see Sec. IV B). This definition is more general because for nonrelativistic quantum tunneling, where symmetric/antisymmetric eigenstate pairs do exist, it reduces to $\Delta E$ used in, e.g., Ref. 18.

\section{MANIFESTATION OF NONHYPERBOLIC CLASSICAL DYNAMICS IN RELATIVISTIC QUANTUM TUNNELING: CLUSTERING OF TUNNELING RATE}

Figure 2 shows the relativistic quantum tunneling rate versus the energy for a mushroom double-well system. Four representative points of different levels of tunneling are identified, which correspond to four characteristically different classical orbits in the mushroom billiard. In particular, the group of points with the highest tunneling rates, e.g., the red dot marked by (a), are associated with the states concentrating 

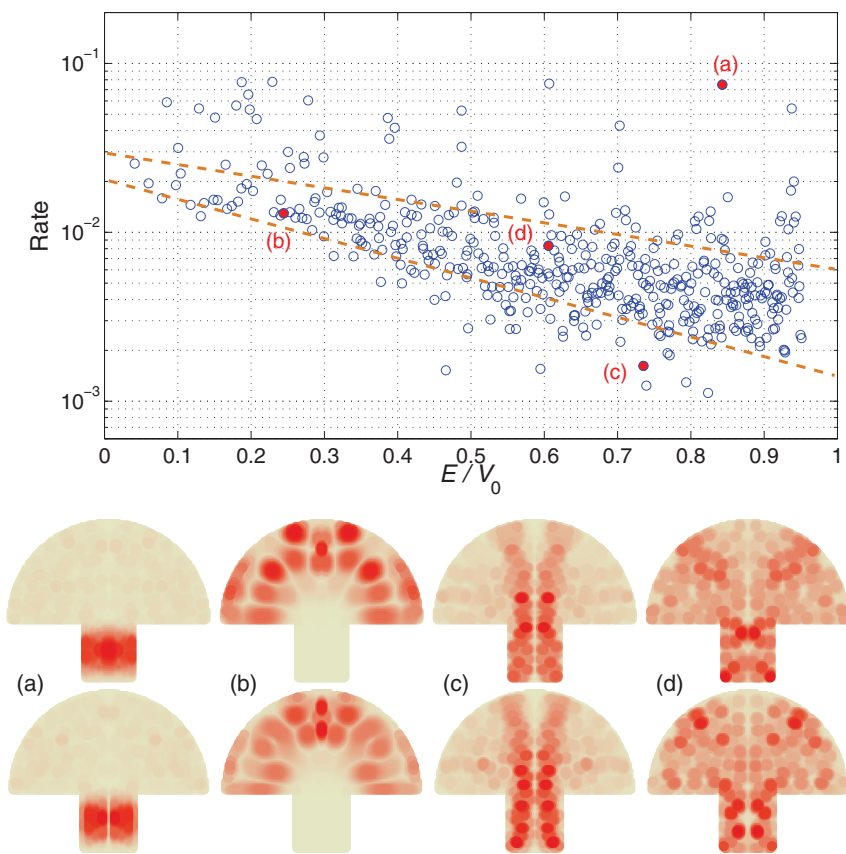

FIG. 2. (Color online) Tunneling rates and eigenstates for a massless Dirac fermion in the mushroom billiard for $r=0.25 R$ and $h=0.6 R$. The height and width of the potential barrier are $V_{0}=60$ and $w=0.15$, respectively. The first and second rows of patterns show the intensity distributions of the $\phi$ and $\chi$ components of the Dirac spinor, respectively. The four red points in the tunneling-rate plot (upper panel) correspond to the patterns for (a) a stable periodic orbit of type-II orbit, (b) a marginally stable periodic orbit of type-I orbit, (c) an orbit with extremely low tunneling rate, and (d) a chaotic orbit of type III.

in the stem of the mushroom, as shown by the associated eigenstates in Fig. 2(a). These states correspond to the classical trajectories of type-II in Fig. 1. Figure 2(b) shows another type of states, those that circle around the mushroom cap, which correspond to the classical trajectory of type-I in Fig. 1. The tunneling rates for this class of states are somewhat intermediate. Figure 2(c) show an eigenstate with relatively low tunneling rate, which is a state localized on the narrow regions near but parallel to the potential barrier, leading to extremely small probability for the state to cross the barrier. No classical periodic orbits can be identified to correspond to such states, due to the potential barrier along the symmetric line of the billiard. Overall, the cases in Figs. 2(a)-2(c) correspond to the integrable component of the classical dynamics. Because of the large variation in the structure of the classical orbits in the integrable component, there is a wide spread in the relativistic quantum tunneling rate. In contrast, eigenstates corresponding to chaotic orbits are "similar" in the sense that they tend to spread over the entire classically allowed region in the billiard domain. As a result, the variation in their tunneling rates is significantly reduced. One such eigenstate is shown in Fig. 2(d). Since the chaotic orbits are uncountable (versus countable orbits belonging to the integrable component), a large number of eigenstates with similar values of the tunneling rate exist, leading to clustering of the rates into a relatively narrow band, as indicated in Fig. 2. The clustering phenomenon persists when the geometry of
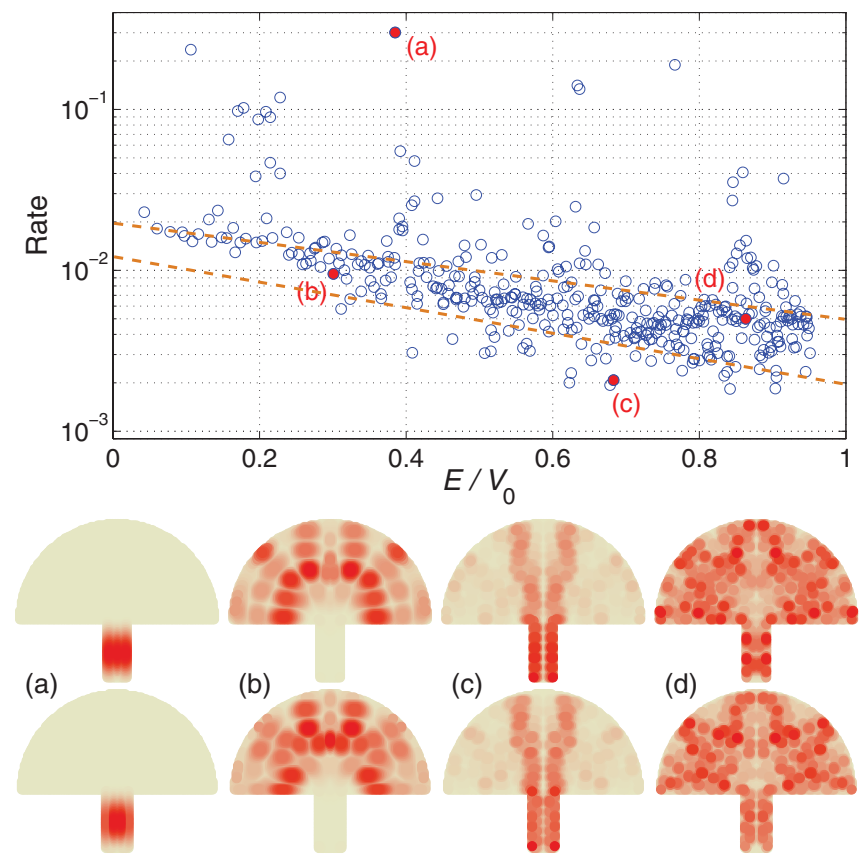

FIG. 3. (Color online) Tunneling rates and representative eigenstates for massless Dirac fermion in mushroom billiard for $r=$ $0.125 R$ and $h=0.6 R$.

the mushroom is varied, such as by decreasing or increasing the size of the rectangular stem relative to the radius of the semicircle, as shown in Figs. 3 and 4, respectively. For those cases, an examination of the corresponding classical phase map reveals that shrinking the width of the mushroom stem makes regular trajectories bouncing between the two walls of the stem more pronounced. This leads accordingly to more
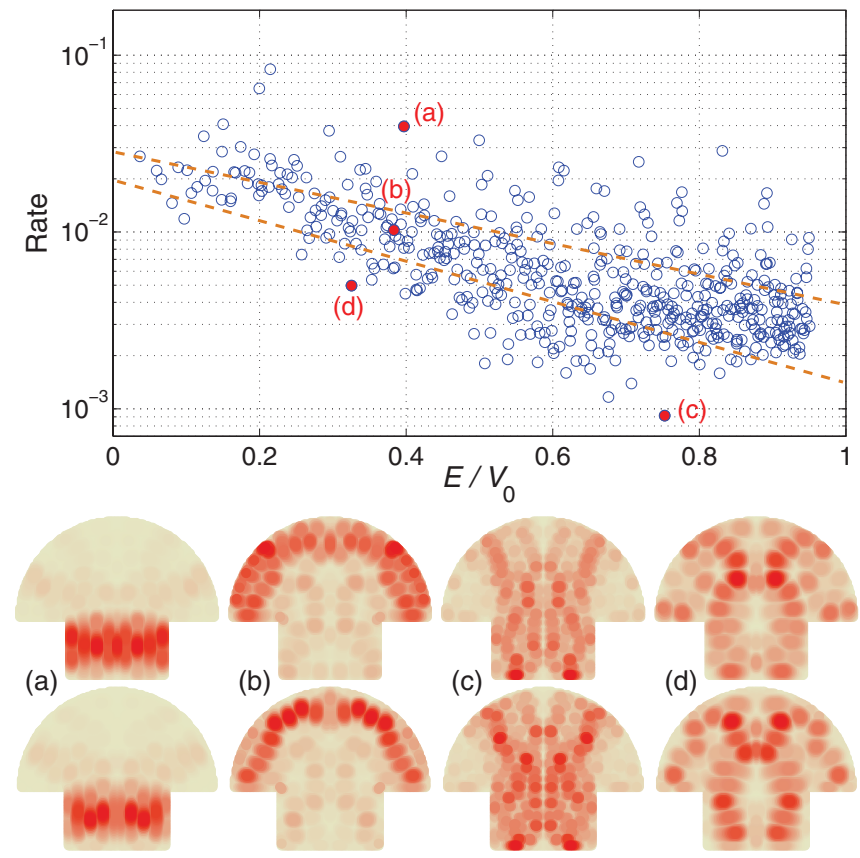

FIG. 4. (Color online) Tunneling rates and representative eigenstates for massless Dirac fermion in mushroom billiard for $r=0.5 R$ and $h=0.6 R$. 

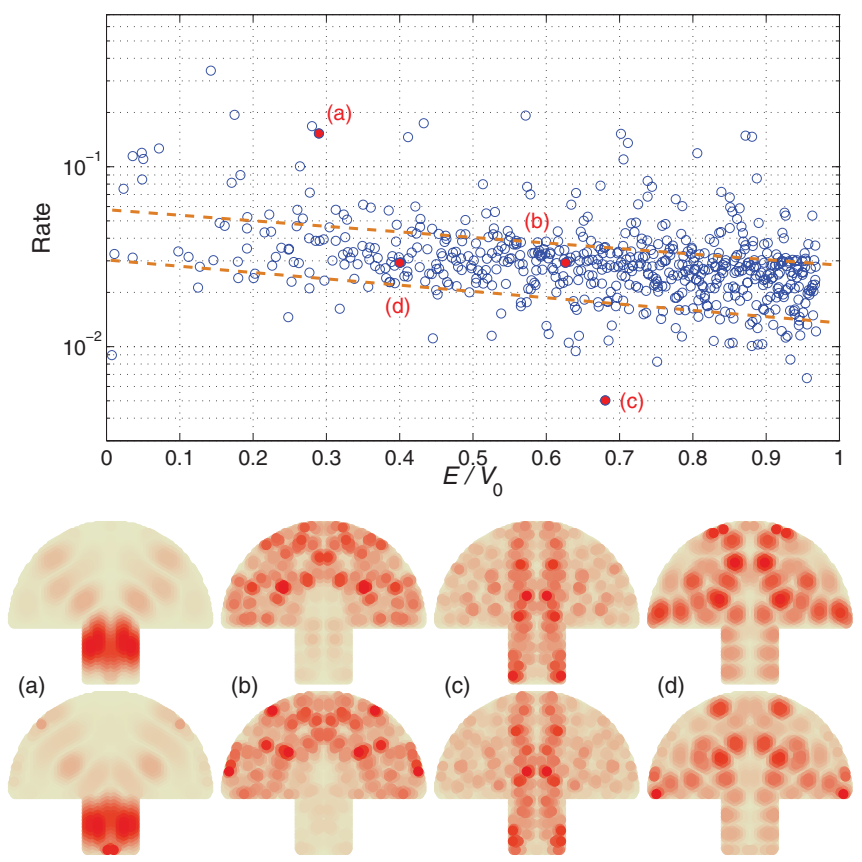

FIG. 5. (Color online) Tunneling rates and representative eigenstates for mushroom billiard made of graphene. The dimensions of the graphene system and the barrier height and width are proportional to those of the Dirac-fermion tunneling system in Fig. 2, but the first and the second rows of patterns represent, instead of Dirac spinors, the $A$ and $B$ atoms in the graphene unit cell. The eigenenergies and eigenstates are calculated by using the standard tight-binding Hamiltonian for the closed graphene system. The legend is the same as that in Fig. 2.

pronounced corresponding relativistic quantum eigenstates. In contrast, enlarging the stem width makes the formation of such "stem states" more difficult, leading to values of the tunneling rate significantly smaller than those in the clustered band.

A similar clustering phenomenon occurs for mushroom billiard of the same geometry but made entirely of graphene, as shown in Fig. 5. It should be noted, however, that the similar tunneling phenomenon presented in the graphene system shall not be mixed with the single massless Dirac fermion system. For applications to graphene specifically, there are recent developments ${ }^{19}$ concerning the proper field theoretical frameworks. There are also semiclassical approaches ${ }^{20}$ to the Dirac equation and its time evolution and time-reversal properties. $^{21}$

The placement of the clustering bands separated by gold dashed lines in Figs. 2-5 are heuristic because, for any given small energy interval, the values of the tunneling rate associated with the chaotic orbits are "embedded" and "mixed" with some values due to the localized states in the integrable component. It is thus difficult to have "clear-cut" values of the chaotic tunneling rate. Nonetheless, the clustering band has two features. First, the width of band appears to increase with energy. This feature is in fact shared by nonrelativistic quantum tunneling in chaotic domains, where it was observed ${ }^{18}$ that the variance of the tunneling rate in a moving window increases with the energy, and this behavior can be explained by using Berry's random-phase approximation ${ }^{22}$ for eigenstates in a fully chaotic domain. Second, for $E \rightarrow 0$, the values of the tunneling rate do not approach zero, in dramatic contrast to nonrelativistic quantum tunneling. This is due to the uniquely relativistic quantum phenomenon of Klein tunneling. ${ }^{23}$

\section{THEORY}

\section{A. General framework}

To develop a theoretical understanding of relativistic quantum tunneling in classically nonhyperbolic systems, we make use of the concept of self-energy from quantum transport theory. ${ }^{24,25}$ The mushroom domain can be regarded as consisting of two closed symmetric wells coupled through the potential barrier along the line of symmetry. For a given state localized in one well, the tunneling rate is effectively the decaying rate of a Dirac fermion into the other well. For convenience, we denote the left well by superscript (1), and the barrier together with the right well by superscript (2), as shown in Fig. 6. The Dirac equation for the whole double-well system can then be written in terms of the Hamiltonians for regions (1) and (2),

$$
\left[\begin{array}{ll}
H_{1} & V_{12} \\
V_{21} & H_{2}
\end{array}\right]\left[\begin{array}{l}
\psi^{(1)} \\
\psi^{(2)}
\end{array}\right]=E\left[\begin{array}{l}
\psi^{(1)} \\
\psi^{(2)}
\end{array}\right],
$$

where $V_{i j}$ are the coupling matrices. If the left well were itself closed, i.e., if it were surrounded by infinitely high potential, the equation would become $H_{1} \psi^{(1)}=E_{1} \psi^{(1)}$. The effect of coupling with the right well can be treated as an equivalent energy term $\Sigma^{R}$, and we have

$$
\left(H_{1}+\Sigma^{R}\right) \psi^{(1)}=E \psi^{(1)},
$$

where

$$
\Sigma^{R}=V_{12} G^{R} V_{21}
$$

is the self-energy for the barrier and the right well, and

$$
G^{R}=\frac{1}{E+i \eta-H_{2}}
$$

is the retarded Green's function. The self-energy term is generally non-Hermitian, leading to complex energy values $E .^{24}$ For each eigenstate of the left well, the energy shift can be obtained through the first-order perturbation theory as

$$
\left\langle\Sigma^{R}\right\rangle=\left\langle\psi^{(1)}\left|\Sigma^{R}\right| \psi^{(1)}\right\rangle,
$$

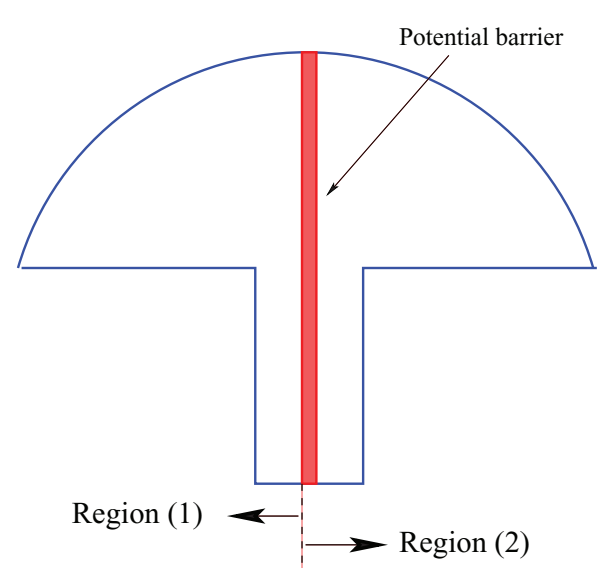

FIG. 6. (Color online) Schematic diagram of the division into subregions in the theoretical calculation of the tunneling rate. 
which is typically complex. The real part of the energy shift changes the oscillating frequency of the corresponding eigenstate, while the imaginary part, denoted by $\gamma$, introduces a decay factor $\exp \left(-c_{0} \gamma t / \hbar\right)$ in the time evolution of the probability, which describes the escaping rate of the Dirac fermion from the left well to the right. Note that, since the whole system is still closed, $\gamma$ only describes the transient event that the particle tunnels from left to right, while recurrences from right to left are ignored. If we let the right well be infinitely long so that there is no reflective wave, the situation becomes a single left well coupled with a semi-infinite electron waveguide (or lead) through the potential barrier, and $\gamma$ will then be the tunneling rate for the single left-well system.

For nonrelativistic quantum tunneling, the self-energy $\Sigma^{R}$ and the complex eigenvalues of the non-Hermitian Hamiltonian $H_{1}+\Sigma^{R}$ can be calculated through the nonequilibrium Green's function approach. Even for a graphene system described by the tight-binding Hamiltonian, this approach can be used. However, for an open fermion system described strictly by the Dirac equation, a Green's function formulation is yet to be developed. Thus for a general geometry such as the nonhyperbolic mushroom billiard or even a domain in which the classical dynamics is fully chaotic, the tunneling rate $\gamma$ cannot be calculated analytically. Nonetheless, for certain integrable geometry, $\gamma$ can be calculated. In the following, we shall first present a one-dimensional analytic example to illustrate the calculation of $\gamma$ and to show the agreement among the three methods to calculate the tunneling rate based on (i) $\Delta T$ [see Eq. (3)], (ii) $\Delta E$, and (iii) $\gamma$. We then present a two-dimensional example to demonstrate the wide spread in the tunneling rate for classically integrable domains.

\section{B. One-dimensional example}

In one spatial dimension where a narrow potential barrier of finite height is placed at the center of an infinite potential well, the tunneling rate can be calculated exactly, with the result that it does not depend on the energy. In particular, consider the system shown in Fig. 7. As we will show, despite its simplicity, the system possesses some important properties of the relativistic quantum tunneling, such as the large tunneling rates for small energies due to the phenomenon of Klein tunneling. We start by solving the one-dimensional

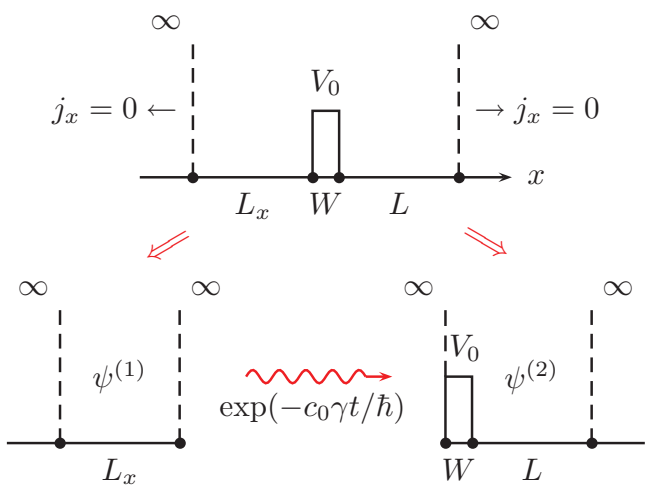

FIG. 7. (Color online) Schematic diagram for one-dimensional tunneling of a massless Dirac fermion.
Dirac equation

$$
\left(-i \hbar v \sigma_{x} \partial_{x}\right) \psi=(E-V) \psi
$$

separately for the two closed systems. For the left well of width $L_{x}, V=0$, so the solution is

$$
\psi_{n}^{(1)}(x)=\frac{1}{\sqrt{L_{x}}} \exp \left(i \frac{\pi}{4}\right)\left[\begin{array}{c}
\cos \left(k_{n} x-\frac{\pi}{4}\right) \\
i \sin \left(k_{n} x-\frac{\pi}{4}\right)
\end{array}\right],
$$

where $k_{n}=(n+1 / 2) \pi / L_{x}$. For the right part, the barrier has width $W$ and $V=V_{0}$, and the right well has width $L$ and $V=0$. We obtain the solution

$$
\psi_{n}^{(2)}(x)= \begin{cases}A_{1} e^{i \kappa_{n} x} u_{+}+A_{2} e^{-i \kappa_{n} x} u_{-}, & \text {barrier } \\ A_{3} e^{i k_{n} x} u_{+}+A_{4} e^{-i k_{n} x} u_{-}, & \text {right well, }\end{cases}
$$

where $k_{n}=E /(\hbar v)=\left[V_{0} W /(\hbar v)+(m+1 / 2) \pi\right] /(L+W)$, $\kappa_{n}=k_{n}-V_{0} /(\hbar v), u_{+}=(1,1)^{T}$ and $u_{-}=(1,-1)^{T}$ are bases for the spinor, and the coefficients $A_{i}$ are determined by the boundary conditions. Note that this is a combined solution for $E<V_{0}$ and $E>V_{0}$. We are only interested in the junction between the left well and the right part where the coupling occurs, and so far we have assumed the $x$ coordinate to be continuous. What we need is a Green's function on a discrete lattice, having lattice points spaced by $a$, between two points along the $x$ axis. We express the energy shift using the discrete lattice

$$
\begin{aligned}
\left\langle\Sigma^{R}\right\rangle= & \psi_{n}^{(1) \dagger}\left(L_{x}-a\right) V_{12} G^{R}\left(L_{x}+a, L_{x}+a\right) V_{21} \\
& \times \psi_{n}^{(1)}\left(L_{x}-a\right),
\end{aligned}
$$

where $x=L_{x}$ is the junction between the left well and the barrier, and the coupling is $V_{12}=V_{21}^{\dagger}=-i \hbar v \sigma_{x}$. It is necessary to compute the Green's function

$$
G^{R}\left(L_{x}+a, L_{x}+a\right)=\sum_{n} \frac{\psi_{n}^{(2)}\left(L_{x}+a\right) \psi_{n}^{(2) \dagger}\left(L_{x}+a\right)}{E+i \eta-\hbar v k_{n}} .
$$

To eliminate any reflection back to the left well, we let the right well be infinitely long, i.e., $L \rightarrow \infty$, so the summation changes into an integral

$$
\sum_{k} \rightarrow \frac{L+W}{\pi} \int d k
$$

Note that $\left|A_{1}\right|^{2}=1 /[4(L+W)]$, which cancels the $L+W$ factor in Eq. (12). The value of the Green's function at $x=$ $L_{x}+a$ becomes

$$
G^{R} \rightarrow \frac{1}{2 \pi} \int_{0}^{\infty} \frac{I_{2}+M_{k}}{E+i \eta-\hbar v k},
$$

where $I_{2}$ is the $2 \times 2$ identity matrix and

$$
M_{k}=\left[\begin{array}{cc}
\sin (2 \kappa a) & i \cos (2 \kappa a) \\
-i \cos (2 \kappa a) & -\sin (2 \kappa a)
\end{array}\right] .
$$

The integral over $I_{2}$ diverges, but the imaginary part of the integral is finite, which eventually contributes to $\gamma$. This imaginary contribution turns out to be $-\hbar v \eta /\left(2 \pi L_{x} E\right)$. To 
calculate the integral for the components of $M_{k}$, we define

$$
\begin{aligned}
& S=\int_{0}^{\infty} d k \frac{\sin (2 \kappa a)}{E+i \eta-\hbar v k}, \\
& C=\int_{0}^{\infty} d k \frac{\cos (2 \kappa a)}{E+i \eta-\hbar v k} .
\end{aligned}
$$

The rate $\gamma$ can be expressed in terms of these integrals as

$$
\begin{aligned}
\gamma= & -\frac{(\hbar v)^{2}}{2 \pi L_{x}}[\cos (2 k a) \operatorname{Im}(C)-\sin (2 k a) \operatorname{Im}(S)] \\
& -\frac{\hbar v \eta}{2 \pi L_{x} E} .
\end{aligned}
$$

Evaluating the integrals $S$ and $C$ and using $E \approx E_{1}=\hbar v k_{n}$, we obtain

$$
\begin{aligned}
\gamma_{n}= & -\frac{\hbar v}{4 \pi L_{x}} \operatorname{Im}\left[2 \operatorname{Ci}\left(-2 k_{n} a-i \delta\right) \cos \left(4 k_{n} a-\frac{2 a V_{0}}{\hbar v}+i \delta\right)\right. \\
& \left.+\left[\pi+2 \operatorname{Si}\left(2 k_{n} a+i \delta\right)\right] \sin \left(4 k_{n} a-\frac{2 a V_{0}}{\hbar v}+i \delta\right)\right] \\
& -\frac{\hbar v \delta}{4 \pi a L_{x} k_{n}},
\end{aligned}
$$

where $\delta=2 a \eta /(\hbar v)$, and $\operatorname{Ci}(x)$ and $\operatorname{Si}(x)$ are cosine and sine integrals defined as

$$
\begin{aligned}
& \operatorname{Ci}(x)=\gamma_{0}+\ln (x)+\int_{0}^{x} \frac{\cos (t)-1}{t} d t, \\
& \operatorname{Si}(x)=\int_{0}^{x} \frac{\sin (t)}{t} d t,
\end{aligned}
$$

where $\gamma_{0}$ is the Euler-Mascheroni constant. When $a$ and $\eta$ are small, we have

$$
\operatorname{Im}\left[\operatorname{Ci}\left(-2 k_{n} a-i \delta\right)\right] \approx-\pi / 2[1+u(\hbar v k-\eta)],
$$

where $u(\cdot)$ is a step function. So for $k>\eta /(\hbar v)$, we obtain a constant expression for $\gamma$,

$$
\gamma=\frac{\hbar v}{2 L_{x}}
$$

the coefficient $c_{0}$ in the exponential-decay factor $\exp \left(-c_{0} \gamma t / \hbar\right)$ can be determined as follows.

Considering the symmetry of the double-well system, we see that the solution $\psi=(\phi, \chi)^{T}$ can be divided into two types: symmetric and antisymmetric. We set the origin at the center of the double well, so for the first component $\phi$, symmetric and antisymmetric solutions require $\phi^{\prime}(0)=0$ and $\phi(0)=0$, respectively. Interestingly, the second component is symmetric when the first is antisymmetric, and vice versa. We name the symmetry type according to the first component, and compute the energy levels for $E<V_{0}$. For symmetric and antisymmetric states, the energy levels are

$$
\begin{aligned}
& E_{S}=\frac{W V_{0} / 2+\left(n_{S}+1 / 4\right) \hbar v \pi}{L_{x}+W / 2}, \\
& E_{A}=\frac{W V_{0} / 2+\left(n_{A}-1 / 4\right) \hbar v \pi}{L_{x}+W / 2},
\end{aligned}
$$

where $n_{S}, n_{A} \in \mathbb{Z}$. Because these energy levels are equally spaced, the energy spacing between symmetric and antisym-

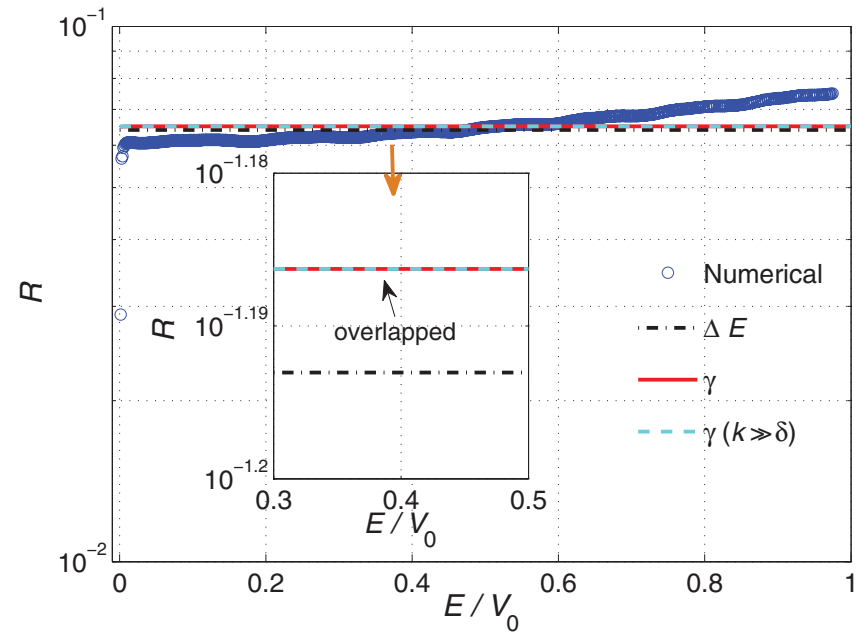

FIG. 8. (Color online) Theoretical tunneling rates compared with numerics for a one-dimensional massless Dirac fermion resonant tunneling system. Two theoretical curves are plotted: $\Delta E$ from the energy spacings between symmetric/antisymmetric eigenstate pairs for the whole double-well system and $\pi \gamma$ from the self-energy method. Inset shows a zoom-in view for $E / V_{0} \in[0.3,0.5]$ where the $\gamma$ curve overlaps with the $\gamma(k \gg \delta)$ curve.

metric states is

$$
\Delta E=\frac{\hbar v \pi}{2 L_{x}+W}
$$

Comparing $\Delta E$ with $\gamma$ and noting that $L_{x} \gg W$, we have the coefficient $c_{0}=\pi$. Figure 8 shows both $\Delta E$ and $\pi \gamma$ in comparison with results from direct numerical evaluation of $\Delta T$ [see Eq. (3)]. We can see that $\pi \gamma$ calculated from the equivalent system using the self-energy method agrees with $\Delta E$ quite well, demonstrating that the tunneling rate is nearly constant for all energies in one dimension. Due to the uniquely relativistic quantum phenomenon of Klein tunneling, we see that the tunneling rate remains large even for $E \rightarrow 0$. This feature absolutely has no classical correspondence.

\section{Resonant tunneling in a two-dimensional Dirac fermion system with circular geometry}

In two dimensions, an analytic solution of the Dirac equation cannot be obtained in general, due to the entanglement of the two Cartesian coordinates in the first-order Dirac equation. The problem is solvable only for certain types of boundary conditions via separation of variables. One particular case is the circular boundary condition. Figure 9 shows a ring with a thin concentric ring-shaped barrier in it, which makes it a double-well system. When all four radii are large, the system is topologically equivalent to a rectangular double well with a periodic boundary condition in one direction. To solve the two-dimensional Dirac equation

$$
(-i \hbar v \sigma \cdot \nabla) \psi=(E-V) \psi
$$

we need to use polar coordinates $(r, \theta)$. Using $\partial_{x} \pm i \partial_{y}=$ $\exp ( \pm i \theta)\left(\partial_{r} \pm i \partial_{\theta} / r\right)$, the general solutions are of the 


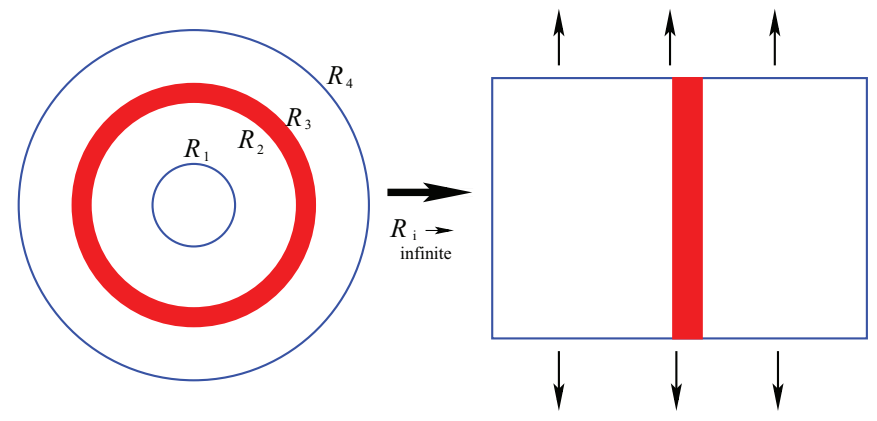

FIG. 9. (Color online) Schematic picture of a solvable 2D geometry. Topologically, for $R_{i} \rightarrow \infty$, the ring is equivalent to a rectangle with periodic boundary conditions in the vertical direction. The shaded regions denote a potential barrier of height $V_{0}$.

following form:

$$
\psi_{n}=e^{i n \theta}\left[\begin{array}{c}
Z_{n}(\lambda r) \\
\operatorname{sgn}(E-V) i e^{i \theta} Z_{n+1}(\lambda r)
\end{array}\right], n= \pm 1, \pm 2, \ldots,
$$

where $Z_{n}(x)=A J_{n}(x)+B Y_{n}(x)$ is a linear combination of the first- and second-kind Bessel functions and $\lambda=$ $|E-V| /(\hbar v)$.

We separate the ring double-well into two parts, the inner ring [denoted by superscript (1)] and the outer part [denoted by superscript (2)] consisting of the barrier ring and the outer ring. To be able to obtain an analytical expression for the energy levels, we use the asymptotic form of the Bessel functions for $x \gg\left|n^{2}-1 / 4\right|$,

$$
\begin{aligned}
& J_{n}(x) \sim \sqrt{\frac{2}{\pi x}} \cos \left(x-\frac{n \pi}{2}-\frac{\pi}{4}\right), \\
& Y_{n}(x) \sim \sqrt{\frac{2}{\pi x}} \sin \left(x-\frac{n \pi}{2}-\frac{\pi}{4}\right) .
\end{aligned}
$$

Applying two boundary conditions at $r=R_{1}, R_{2}$ and normalization, we have for large radii, the solution for the inner ring:

$$
\psi_{m, n}^{(1)}=N \frac{1}{\sqrt{r}} e^{i n \theta}\left\{\begin{array}{c}
\sin \left[k_{m}\left(r-R_{2}\right)+3 \pi / 4\right] \\
-i e^{i \theta} \cos \left[k_{m}\left(r-R_{2}\right)+3 \pi / 4\right]
\end{array}\right\},
$$

where

$$
k_{m}=\frac{(m+1 / 2) \pi}{R_{2}-R_{1}}
$$

$m=0,1,2, \ldots$, and $N=\left[2 \pi\left(R_{2}-R_{1}\right)\right]^{-1 / 2}$. Note that the eigenenergy $\hbar v k_{m}$ does not depend on the angular quantum number $n$, meaning that all different angular modes are degenerate for a single energy level. This happens when we let the ring be infinitely large, where the radial functions take the trigonometric form and the variation of the functions becomes periodic so that the energy levels are equally spaced. However, if we were to use the original radial solution, the energy levels can be found through the zeros of the integer-order Bessel functions, and thus are spaced with a decreasing spacing. In that case, because the different integer-order Bessel functions differ in zeros, the eigenenergies are nondegenerate. For the outer part, the solutions combining $E<V_{0}$ and $E>V_{0}$ are

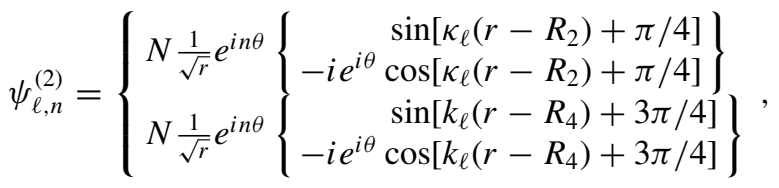

where

$$
k_{\ell}=\left[\left(R_{3}-R_{2}\right) V_{0} /(\hbar v)+(\ell+1 / 2) \pi\right] /\left(R_{4}-R_{2}\right)
$$

and $\kappa_{\ell}=k_{\ell}-V_{0} /(\hbar v)$. Similar to one dimension, the Green's function at the coupling boundary $R_{2}+a$ is

$$
\left.G^{R}\left(r, \theta ; r, \theta^{\prime}\right)\right|_{r=R_{2}+a}=\sum_{n} \sum_{\ell} \frac{\psi_{\ell, n}^{(2)}(r, \theta) \psi_{\ell, n}^{(2) \dagger}\left(r, \theta^{\prime}\right)}{E+i \eta-\hbar v k_{\ell}} .
$$

Letting the outer boundary be infinite so the summation over $k_{\ell}$ turns into an integration, and we have

$$
G^{R} \rightarrow \sum_{n} \frac{e^{i n\left(\theta-\theta^{\prime}\right)}}{(2 \pi)^{2} R_{2}} \int_{0}^{\infty} d k \frac{M_{k}^{\prime}}{E+i \eta-\hbar v k},
$$

where

$$
M_{k}^{\prime}=\left[\begin{array}{lc}
1+\sin (2 \kappa a) & i e^{-i \theta^{\prime}} \cos (2 \kappa a) \\
-i e^{i \theta} \cos (2 \kappa a) & e^{i\left(\theta-\theta^{\prime}\right)}[1-\sin (2 \kappa a)]
\end{array}\right] .
$$

The energy shift is given by

$$
\left\langle\Sigma^{R}\right\rangle_{m}=\int d \theta \int d \theta^{\prime} \psi_{m, j}^{(1) \dagger}(\theta) V_{12} G^{R}\left(\theta ; \theta^{\prime}\right) V_{21} \psi_{m, j}^{(1)}\left(\theta^{\prime}\right),
$$

where $\psi_{m, j}^{(1)}\left(\theta^{\prime}\right)$ is computed at $r=R_{2}-a$, and the coupling matrix for two dimensions is obtained as follows. Using

$$
\boldsymbol{\sigma} \cdot \nabla=\left[\begin{array}{cc}
0 & e^{-i \theta} \\
e^{i \theta} & 0
\end{array}\right] \partial_{r}+\left[\begin{array}{cc}
0 & -i e^{-i \theta} \\
i e^{i \theta} & 0
\end{array}\right] \frac{\partial_{\theta}}{r},
$$

we have the coupling matrix for $r$. Carrying out the integration over the coordinates $\theta$ and $\theta^{\prime}$,

$$
\int_{0}^{2 \pi} d \theta \int_{0}^{2 \pi} d \theta^{\prime} e^{i(j-n)\left(\theta-\theta^{\prime}\right)}=(2 \pi)^{2} \delta_{j n},
$$

we see that the summation over $n$ can be simplified. The final expression for rate $\gamma$ is exactly the same as in one dimension, i.e., Eqs. (17) and (18). The reason for the same rate $\gamma$ as in one dimension is largely due to the degeneracy of the angular modes. Because all tangent modes are degenerate for one specific energy level that depends on the radial function only, one cannot separate this system from a true one-dimensional system.

While the two-dimensional solution we have presented here is a special case where we have used the large $r$ asymptotic form of the radial functions, the general solution for circular boundaries is different because, for a certain energy level, the degenerate angular modes require an infinite $R_{i}$ condition. However, for finite rings, different angular modes are associated with different energy levels. Therefore the tunneling rates for finite rings have a wide spread instead of concentrating on a single straight line. In order to obtain the rate $\gamma$ for finite-size rings, we must solve the eigenstates numerically. Using the general solution for finite inner ring, 


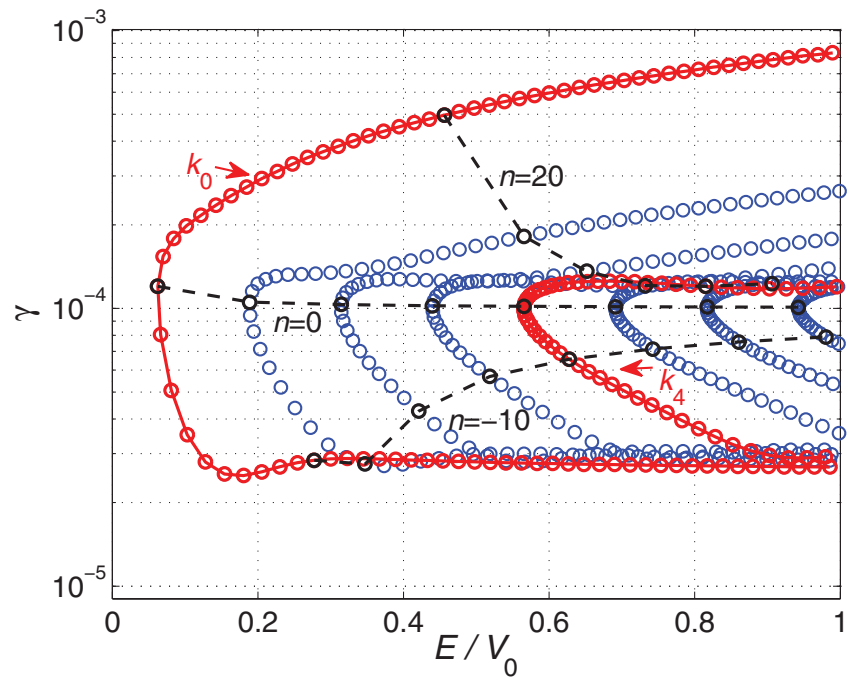

FIG. 10. (Color online) Theoretical rate $\gamma$ for a ring-type of resonant tunneling system, where $n$ denotes the angular quantum number, and $k_{i}$ is the $i$ th energy level for all $n$. The eigenstates of the inner ring are found numerically, while the Green's function values for the outer part are calculated analytically. The radii and potential height are $R_{1}=5, R_{2}=10, R_{3}=10.2, R_{4} \rightarrow \infty$, and $V_{0}=5$.

Eq. (21), the normalization of the eigenstates, and the boundary conditions $Z_{n+1}\left(k_{m} R_{i}\right) / Z_{n}\left(k_{m} R_{i}\right)= \pm 1$ at $r=R_{i}, i=1,2$, we can find the roots $k_{m}$ for each angular mode $n$ numerically. To eliminate reflection, we set $R_{4}$ to infinite. This means we need to sum over infinite terms of $k_{\ell}$ in Eq. (26). A strategy to deal with the difficulty is to set a threshold $k_{\max }$ in the summation, and the criterion for choosing such $k_{\max }$ is the quality of the orthogonality,

$$
\sum_{\left\{\ell: k_{\ell}<k_{\max }\right\}, n} \psi_{\ell, n}^{(2)}(\mathbf{r}) \psi_{\ell, n}^{(2) \dagger}\left(\mathbf{r}^{\prime}\right) \approx I_{2} \delta^{2}\left(\mathbf{r}-\mathbf{r}^{\prime}\right) .
$$

However, as the number of eigenstates $\ell$ below $k_{\max }$ increases, the computation burden increases as $\ell^{2}$. For fast computations, we consider two approximate results for the outer part: one that uses the analytical solution of Green's function in Eq. (27), and the other that uses a relatively large outer ring to numerically calculate the rates.

Some representative $\gamma$ values are shown in Fig. 10. We see that the tunneling rates associated with angular modes for the same energy level (e.g., $k_{0}$ and $k_{4}$ in Fig. 10) separate and spread into a wide range, instead of clustering together as a single point, which is the case in one dimension and two-dimensional rings with all infinite radii. An alternative approach is to choose a large but finite value of $R_{4}$ and compute the tunneling rate $\gamma$ numerically. Again, we find a wide spread in $\gamma$, and there are extremely small values of $\gamma$, corresponding to survival states from the opening process of the originally closed left well (inner ring) when a finite potential barrier is introduced into the system.

\section{CONCLUSIONS}

The question we have addressed in this paper is fundamental to physics: what are the relativistic quantum manifestations of nonhyperbolic chaotic systems? In the classical limit, such systems are characterized by the coexistence of chaos and KAM tori in the phase space, or a mixed phase space. To explore and understand the quantum behaviors of such systems is challenging, due to the fact that they are neither completely chaotic or integrable. Particularly, for an integrable system, analytic theory is often available. For a fully chaotic or hyperbolic system, frameworks such as the random-matrix theory or the random-wave approximation can be employed. In the spectrum of Hamiltonian systems, nonhyperbolic systems are generic and typical because they lie in between the two extreme cases of integrable and hyperbolic systems. As such, the standard theoretical methods are often not applicable. In the past, the problem has been addressed to certain extent but exclusively in the realm of nonrelativistic quantum mechanics governed by the Schrödinger equation. To explore the relativistic quantum phenomena associated with classically nonhyperbolic chaotic systems is of tremendous interest due to their high relevance to graphene systems.

We have investigated the relativistic quantum manifestations of classical nonhyperbolic dynamics by using the setting of resonant tunneling of a Dirac fermion across a potential barrier. A clustering phenomenon has been uncovered, where the quantum tunneling rate as a function of the energy tends to focus in a narrow band due to the chaotic component in the classical phase space. Relatively fewer values of the tunneling rate spread outside the band due to the integrable component, which can be understood by explicit solutions of the Dirac equation in some special geometrical domains. Due to the rapid development of relativistic quantum solid-state devices in recent years, such as graphene systems, it would be interesting and rewarding to extend the study of relativistic quantum manifestations of nonhyperbolic dynamics for Dirac fermions to other problems as well.

\section{ACKNOWLEDGMENT}

This work was supported by AFOSR under Grant No. FA9550-12-1-0095 and by ONR under Grant No. N0001408-1-0627. LH was also supported by NSFC under Grant No. 11005053.

\footnotetext{
*Ying-Cheng.Lai@asu.edu

${ }^{1}$ H. J. Stöckmann, Quantum Chaos: An Introduction (Cambridge University Press, Cambridge, England, 1999).

${ }^{2}$ A. J. Lichtenberg and M. A. Lieberman, Regular and Chaotic Dynamics, Applied Mathematical Sciences Vol. 38, 2nd ed. (Springer-Verlag, New York, 1992).
}

${ }^{3}$ E. Ott, Chaos in Dynamical Systems, 2nd ed. (Cambridge University Press, Cambridge, UK, 2002).

${ }^{4}$ Y.-C. Lai and T. Tél, Transient Chaos: Complex Dynamics on FiniteTime Scales (Springer, New York, 2011).

${ }^{5}$ Y.-C. Lai, R. Blümel, E. Ott, and C. Grebogi, Phys. Rev. Lett. 68, 3491 (1992). 
${ }^{6}$ R. Ketzmerick, Phys. Rev. B 54, 10841 (1996); A. S. Sachrajda, R. Ketzmerick, C. Gould, Y. Feng, P. J. Kelly, A. Delage, and Z. Wasilewski, Phys. Rev. Lett. 80, 1948 (1998); B. Huckestein, R. Ketzmerick, and C. H. Lewenkopf, ibid. 84, 5504 (2000); G. Casati, I. Guarneri, and G. Maspero, ibid. 84, 63 (2000); R. Crook, C. G. Smith, A. C. Graham, I. Farrer, H. E. Beere, and D. A. Ritchie, ibid. 91, 246803 (2003).

${ }^{7}$ K. S. Novoselov, A. K. Geim, S. V. Morozov, D. Jiang, Y. Zhang, S. V. Dubonos, I. V. Grigorieva, and A. A. Firsov, Science 306, 666 (2004); C. Berger, Z. Song, T. Li, X. Li, A. Y. Ogbazghi, R. Feng, Z. Dai, A. N. Marchenkov, E. H. Conrad, P. N. First, and W. A. de Heer, J. Phys. Chem. B 108, 19912 (2004); K. S. Novoselov, A. K. Geim, S. V. Morozov, D. Jiang, M. I. Katsnelson, I. V. Grigorieva, S. V. Dubonos, and A. A. Firsov, Nature (London) 438, 197 (2005); Y. Zhang, Y.-W. Tan, H. L. Stormer, and P. Kim, ibid. 438, 201 (2005).

${ }^{8}$ A. H. Castro Neto, F. Guinea, N. M. R. Peres, K. S. Novoselov, and A. K. Geim, Rev. Mod. Phys. 81, 109 (2009); N. M. R. Peres, ibid. 82, 2673 (2010); S. Das Sarma, S. Adam, E. H. Hwang, and E. Rossi, ibid. 83, 407 (2011).

${ }^{9}$ L. Huang, Y.-C. Lai, D. K. Ferry, S. M. Goodnick, and R. Akis, Phys. Rev. Lett. 103, 054101 (2009); L. Huang, Y.-C. Lai, and C. Grebogi, Phys. Rev. E 81, 055203(R) (2010); R. Yang, L. Huang, Y.-C. Lai, and L. M. Pecora, Appl. Phys. Lett. 100, 093105 (2012).

${ }^{10}$ M. V. Berry and R. J. Mondragon, Proc. R. Soc. London A 412, 53 (1987).

${ }^{11}$ H.-Y. Xu, L. Huang, Y.-C. Lai, and C. Grebogi, Phys. Rev. Lett. 110, 064102 (2013).

${ }^{12}$ X. Ni, L. Huang, Y.-C. Lai, and L. M. Pecora, Europhys. Lett. 98, 50007 (2012).
${ }^{13}$ L. Bunimovich, Chaos 11, 802 (2001).

${ }^{14}$ S. Löck, A. Bäcker, R. Ketzmerick, and P. Schlagheck, Phys. Rev. Lett. 104, 114101 (2010); A. Bäcker, R. Ketzmerick, and S. Löck, Phys. Rev. E 82, 056208 (2010); N. Mertig, S. Löck, A. Bäcker, R. Ketzmerick, and A. Shudo, Europhys. Lett. 102, 10005 (2013).

${ }^{15}$ A. Bäcker, R. Ketzmerick, S. Löck, M. Robnik, G. Vidmar, R. Höhmann, U. Kuhl, and H.-J. Stöckmann, Phys. Rev. Lett. 100, 174103 (2008)

${ }^{16}$ T. D. Newton and E. P. Wigner, Rev. Mod. Phys. 21, 400 (1949).

${ }^{17}$ X. Ni, L. Huang, Y.-C. Lai, and C. Grebogi, Phys. Rev. E 86, 016702 (2012).

${ }^{18}$ L. M. Pecora, H. Lee, D.-H. Wu, T. Antonsen, M.-J. Lee, and E. Ott, Phys. Rev. E 83, 065201(R) (2011); M.-J. Lee, T. M. Antonsen, E. Ott, and L. M. Pecora, ibid. 86, 056212 (2012).

${ }^{19}$ I. V. Fialkovsky and D. V. Vassilevich, Int. J. Mod. Phys. A 27, 1260007 (2012); V. P. Gusynin, S. G. Sharapov, and J. P. Carbotte, Int. J. Mod. Phys. B 21, 4611 (2007); C. G. Beneventano and E. M. Santangelo, Int. J. Mod. Phys. Conf. Ser. 14, 240 (2012).

${ }^{20}$ J. Bolte and S. Keppeler, Phys. Rev. Lett. 81, 1987 (1999); Ann. Phys. 274, 125 (1999); J. Phys. A: Math. Gen. 32, 8863 (1999).

${ }^{21}$ J. Bolte and J. Harrison, J. Phys. A: Math. Gen. 36, 2747 (2003).

${ }^{22}$ M. V. Berry, J. Phys. A 10, 2083 (1977).

${ }^{23}$ P. Strange, Relativistic Quantum Mechanics with Applications in Condensed Matter Physics and Atomic Physics (Cambridge University Press, Cambridge, UK, 1998).

${ }^{24}$ S. Datta, Electronic Transport in Mesoscopic Systems (Cambridge University Press, Cambridge, UK, 1995).

${ }^{25}$ R. Yang, L. Huang, Y.-C. Lai, and C. Grebogi, Europhys. Lett. 94, 40004 (2011). 